\title{
A Novel Two-step Distance Protection for Flexible HVDC Transmission Lines
}

\author{
Zeya Fang ${ }^{1}$, Minghao Wen ${ }^{2}$, Junchao Zheng ${ }^{3}$ and Minghao Wen ${ }^{1 *}$ \\ ${ }^{1}$ State Key Laboratory of Advanced Electromagnetic Engineering and Technology, Huazhong University of Science and Technology, \\ Wuhan, Hubei, 430074, China \\ ${ }^{2}$ State Key Laboratory of Advanced Electromagnetic Engineering and Technology, Huazhong University of Science and Technology, \\ Wuhan, Hubei, 430074, China \\ ${ }^{3}$ State Grid Jiangsu Electric Power Research Institute, Nanjing, Jiangsu, 210036, China
}

\begin{abstract}
DC line fault is one of the key problems that must be solved in a flexible HVDC system. During quite a long time between the existing main protection and backup protection of the HVDC line, there is no line protection method to detect the fault, which may lead the protection at the AC side to act before the backup protection of the DC line. To solve the problem, a novel two-step distance protection for flexible HVDC lines is proposed in this manuscript. Firstly, based on the uniform distributed parameter model, the equivalent lumped parameter model of the HVDC transmission line at low frequency is analyzed. Secondly, according to the time domain differential equation and the least squares algorithm, novel distance protection based on the iterative calculation of fault distance is proposed, which can eliminate the influence of distributed capacitive current and improve the precision of calculation. To improve the rapidity and reliability of the distance protection, low pass filters with two different cut-off frequencies are used to process the electrical quantities. Finally, simulation results show that the proposed distance protection can respond to metallic poleto-ground faults and pole-to-pole faults rapidly and reliably.
\end{abstract}

\section{Introduction}

The flexible HVDC power transmission technology based on the modular multilevel converter (MMC) has a wide application prospect in the fields of large-scale distributed power grid interconnection, asynchronous grid interconnection, and urban HVDC transmission and distribution[1-3]. Due to the complexity of the transmission environment and the increase of transmission distance and capacity, overhead lines are often used in flexible HVDC systems for large-scale power transmission[4]. The HVDC transmission line can be thousands of kilometers long and the climate condition along the line is complex, which makes the probability of HVDC line faults high. The statistical data show that the number of forced outages caused by HVDC transmission line fault accounted for $36.8 \%$ of the total number from 2006 to 2012[5]. Therefore, the operation performance of HVDC line protection is of great significance to the security and stability of HVDC transmission systems.

The existing flexible HVDC transmission lines mostly adopt the technology routes of ABB and SIEMENS. The main protection is configured with the traveling-wave protection and the differential under-voltage protection. The basic principle of the main protection is to identify faults through the amplitude of traveling-wave and the rate of change of voltage[6]. However, the traveling-wave protection and differential under-voltage protection have low sensitivity with a high sampling rate, and difficult to detect faults encountering noise disturbance; the backup protection mainly uses current differential protection. To avoid the influence of distributed capacitive current, the current differential protection often adopts a large time delay, which will be too long for the flexible HVDC systems. Therefore, during the long time between the existing main protection and backup protection of the HVDC line, there is no line protection method to detect faults. To identify the line faults quickly and reliably, it is urgent to propose new protection methods.

In the traditional $\mathrm{AC}$ system, distance protection has the advantages of sufficient theoretical basis, low sampling rate, and not being affected by the operation conditions of the system. In recent years, many works and algorithms have aimed to apply distance protection in HVDC systems. In [7], the fault distance is calculated by solving the differential equations based on the distributed parameter line model. In [8], more accurate distance protection for the HVDC line is proposed, which uses a frequency-dependent line model and has higher accuracy. However, the above two methods are based on distributed parameter line models, which require high sampling frequency and a large amount of calculating. In [9], a distance protection algorithm based on the RL line model is proposed, which can detect the internal fault through the polarity of the ratio of the compensation voltage at the setpoint and the measured voltage. Reference [10]

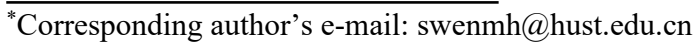


considers the influence of line parameter error for distance protection criterion and proposes that the criterion extremum of the ratio can be used to identify the internal and external faults, which will avoid mal-operation. However, the above two methods ignore the impact of distributed capacitive current on the calculation results.

This paper proposes a novel two-step distance protection scheme based on the iterative calculation of fault distance, which can eliminate the influence of distributed capacitive current and improve the precision of calculation. Simulation results show that the proposed distance protection can rapidly and reliably respond to metallic pole-to-ground faults and pole-to-pole faults.

\section{Research on the equivalent lumped parameter model of HVDC transmission lines}

By solving the equation of the uniform distributed parameter model in the frequency domain, the relationship between $U_{\mathrm{M}}(\mathrm{s}), I_{\mathrm{M}}(\mathrm{s}), U_{\mathrm{N}}(\mathrm{s}), I_{\mathrm{N}}(\mathrm{s})$ at the end of the line can be described by $(1) \sim(2)$.

$$
\begin{gathered}
{\left[\begin{array}{c}
\dot{U}_{M}(s) \\
\dot{I}_{M}(s)
\end{array}\right]=} \\
\left\{\begin{array}{cc}
\cosh (\gamma(s) l) & Z_{c}(s) \sinh (\gamma(s) l) \\
\sinh (\gamma(s) l) / Z_{c}(s) & \cosh (\gamma(s) l)
\end{array}\right]\left[\begin{array}{l}
\dot{U}_{N}(\mathrm{~s}) \\
\dot{I}_{N}(s)
\end{array}\right] \\
\left\{\begin{array}{l}
\gamma(s)=\sqrt{(R+s L)(G+s C)} \\
Z_{c}(s)=\sqrt{(R+s L) /(G+s C)}
\end{array}\right.
\end{gathered}
$$

Where $l$ represents the length of the line; $\gamma$ and $Z_{\mathrm{c}}$ are the propagation constant and characteristic impedance of the line; $R$ and $L$ are the resistance and inductance of the line in per unit length; $G$ and $C$ are the shunt conductance and shunt capacitance of the line in per unit length.

According to the relationship shown in (1), the twoport equivalent circuit diagram shown in Figure 1 can be obtained.

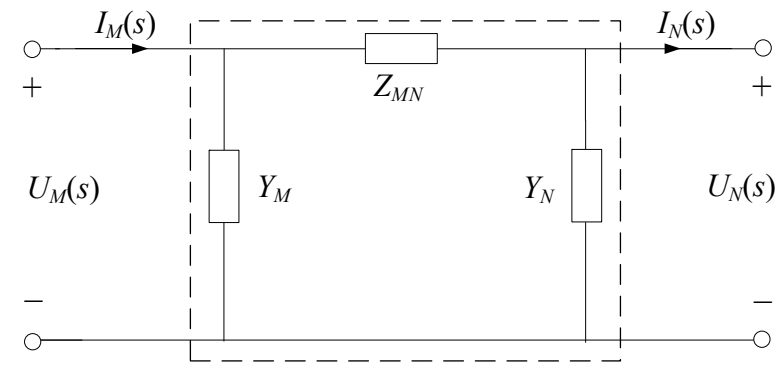

Figure 1. The equivalent circuit diagram for the two-port network of DC transmission line.

When the shunt conductance $G$ can be ignored and $\gamma(\mathrm{s}) l$ is small enough, equation (3) (4) can be deduced:

$$
\begin{aligned}
& \operatorname{sh}(\gamma(s) l) \approx \operatorname{th}(\gamma(s) l) \approx \gamma(s) l \\
& \left\{\begin{array}{c}
Z_{M N}=Z_{c}(s) \operatorname{sh}(\gamma(s) l) \approx(R+j \omega L) l \\
Y_{M}=Y_{N}=\operatorname{th}(\gamma(s) l / 2) / Z_{c}(s) \approx j \omega C l / 2
\end{array}\right.
\end{aligned}
$$

As shown in (4), if the frequency $f$ is low so that $\gamma(\mathrm{s}) l$ is small enough, the DC line can be simplified from the distributed parameter line model to the $\pi$-model.
Therefore, after the voltages and currents are processed by a low-pass filter with sufficiently low cut-off frequency, the line model from both ends of the DC line to the fault point can be approximately equivalent to the $\pi$-model circuit.

\section{Calculation and analysis of fault distance for HVDC transmission lines}

\subsection{Calculation of fault distance for the internal pole-to-ground fault}

When the internal pole-to-ground fault occurs at the DC line, the equivalent $\pi$-model circuit from the $M$ side to the fault point at low frequency can be shown in Figure 2.

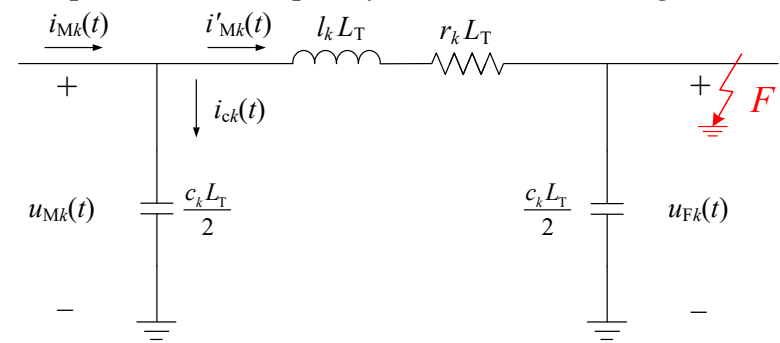

Figure 2. The equivalence circuit of DC lines when internal faults occur

According to Figure 2, the following expression can be obtained.

$$
\begin{aligned}
& u_{\mathrm{Mp}}(t)=\left[r_{s} \cdot i_{\mathrm{Mp}}^{\prime}(t)+l_{s} \frac{\mathrm{d} i_{\mathrm{Mp}}^{\prime}(t)}{\mathrm{d} t}+r_{m} \cdot i_{\mathrm{Mn}}^{\prime}(t)+l_{m} \frac{\mathrm{d} i_{\mathrm{Mn}}^{\prime}(t)}{\mathrm{d} t}\right] \cdot L_{\mathrm{T}}+u_{\mathrm{Fp}}(t) \\
& u_{\mathrm{Mn}}(t)=\left[r_{s} \cdot i_{\mathrm{Mn}}^{\prime}(t)+l_{s} \frac{\mathrm{d} i_{\mathrm{Mn}}^{\prime}(t)}{\mathrm{d} t}+r_{m} \cdot i_{\mathrm{Mp}}^{\prime}(t)+l_{m} \frac{\mathrm{d} i_{\mathrm{Mp}}^{\prime}(t)}{\mathrm{d} t}\right] \cdot L_{\mathrm{T}}+u_{\mathrm{Fn}}(t)
\end{aligned}
$$

Where $u_{\mathrm{Mp}}(\mathrm{t})$ and $u_{\mathrm{Mn}}(\mathrm{t})$ are the instantaneous filtered voltage of the positive pole and the negative pole; $i_{\mathrm{Mp}}^{\prime}(\mathrm{t})$ and $i_{\mathrm{Mn}}^{\prime}(\mathrm{t})$ are the instantaneous filtered current through the line impedance; $r_{\mathrm{s}}$ and $r_{\mathrm{m}}$ are the self-resistance and mutual-resistance of the line in per unit length; $l_{\mathrm{s}}$ and $l_{\mathrm{m}}$ are the self-inductance and mutual-inductance of the line in per unit length; $L_{\mathrm{T}}$ is the fault distance; $u_{\mathrm{Fp}}(\mathrm{t})$ and $u_{\mathrm{Fn}}(\mathrm{t})$ are the instantaneous voltage of the positive pole and the negative pole at the fault point. The relationship between $i_{M p}^{\prime}(\mathrm{t}), i_{M n}^{\prime}(\mathrm{t})$ and $i_{M p}(\mathrm{t}), i_{M n}(\mathrm{t})$ could be calculated by $(6)$ :

$$
\left\{\begin{array}{l}
i_{\mathrm{Mp}}^{\prime}(t)=i_{\mathrm{Mp}}(t)-i_{\mathrm{Mpc}}(t) \\
i_{\mathrm{Mn}}^{\prime}(t)=i_{\mathrm{Mn}}(t)-i_{\mathrm{Mnc}}(t)
\end{array}\right.
$$

Where $i_{\mathrm{Mpc}}(\mathrm{t})$ and $i_{\mathrm{Mnc}}(\mathrm{t})$ represent the shunt capacitive current of the positive pole and the negative pole. Take the metallic positive pole-to-ground (PTG) fault as an example for analysis, and the analysis for the negative pole-to-ground(NTG) fault is similar. When an internal metallic PTG fault occurs, since the currents $i_{\mathrm{Mp}}^{\prime}(\mathrm{t})$ and $i_{M n}^{\prime}(\mathrm{t})$ are unknown, the idea of iterative calculation can be applied. Firstly, use the measured current $i_{\mathrm{Mp}}(\mathrm{t})$ and $i_{\mathrm{Mn}}(\mathrm{t})$ to replace $i_{\mathrm{Mp}}^{\prime}(\mathrm{t})$ and $i_{\mathrm{Mn}}^{\prime}(\mathrm{t})$ to calculate $L_{\mathrm{T}(1)}$ :

$$
u_{\mathrm{Mp}}(t)=\left[r_{s} \cdot i_{\mathrm{Mp}}(t)+l_{s} \frac{\mathrm{d} i_{\mathrm{Mp}}(t)}{\mathrm{d} t}+r_{m} \cdot i_{\mathrm{Mn}}(t)+l_{m} \frac{\mathrm{d} i_{\mathrm{Mn}}(t)}{\mathrm{d} t}\right] \cdot L_{\mathrm{T}(1)}
$$


Based on several consecutive measured voltage and current values, the initial iterative value of fault distance $L_{\mathrm{T}(1)}$ can be calculated by the least squares algorithm. Using this initial value $L_{\mathrm{T}(1)}$, the shunt capacitive current $i_{\text {Mpc }}(\mathrm{t})$ and $i_{\text {Mnc }}(\mathrm{t})$ can be calculated by $(8)$ :

$$
\left\{\begin{array}{l}
i_{\mathrm{Mpc}(1)}(t)=\frac{L_{\mathrm{T}(1)}}{2}\left[c_{s} \frac{\mathrm{d} u_{\mathrm{Mp}}(t)}{\mathrm{d} t}+c_{m} \frac{\mathrm{d} u_{\mathrm{Mn}}(t)}{\mathrm{d} t}\right] \\
i_{\mathrm{Mnc}(1)}(t)=\frac{L_{\mathrm{T}(1)}}{2}\left[c_{s} \frac{\mathrm{d} u_{\mathrm{Mn}}(t)}{\mathrm{d} t}+c_{m} \frac{\mathrm{d} u_{\mathrm{Mp}}(t)}{\mathrm{d} t}\right]
\end{array}\right.
$$

$c_{\mathrm{s}}$ and $c_{\mathrm{m}}$ are the self-capacitance and mutualcapacitance of the line in per unit length. According to (6) and (8), the instantaneous current through the line impedance can be expressed in (9):

$$
\left\{\begin{array}{l}
i_{\mathrm{Mp}(1)}^{\prime}(t)=i_{\mathrm{Mp}}(t)-i_{\mathrm{Mpc}(1)}(t) \\
i_{\mathrm{Mn}(1)}^{\prime}(t)=i_{\mathrm{Mn}}(t)-i_{\mathrm{Mnc}(1)}(t)
\end{array}\right.
$$

Combining (5) and (9), the second iteration value of fault distance $L_{\mathrm{T}(2)}$ can be calculated by using the least squares algorithm. After obtaining $L_{\mathrm{T}(2)}$, repeat the above calculation and finally get the $q$-th iteration value of fault distance $L_{\mathrm{T}(\mathrm{q})}$.

\subsection{Calculation of fault distance for the internal pole-to-pole fault}

When the pole-to-pole (PTP) fault occurs inside the DC line, the expression (5) is still satisfied. Due to the transition resistance of the PTP fault is usually small, the instantaneous voltage values at the fault point of the positive pole and the negative pole are approximately equal, so the following expression can be obtained:

$$
u_{\mathrm{M} 1}(t)=\left[r_{1} \cdot i_{\mathrm{M} 1}^{\prime}(t)+l_{1} \frac{\mathrm{d} i_{\mathrm{M} 1}^{\prime}(t)}{\mathrm{d} t}\right] \cdot L_{\mathrm{T}}
$$

Where $u_{\mathrm{M} 1}(\mathrm{t})$ is the 1 -mode voltage at the relay protection device, $i_{M 1}^{\prime}(\mathrm{t})$ is the 1-mode current through the line impedance, $r_{1}$ and $l_{1}$ are the 1-mode resistance and 1mode inductance of the line in per unit length. Similarly, referring to the iterative calculation method in subsection 3.1, the fault distance $L_{\mathrm{T}}$ can be calculated by using the 1mode voltage and current When the PTP fault occurs.

\subsection{Calculation of fault distance for the external fault}

When the external fault occurs, since there is no zerocrossing point of the voltage inside the DC line, the voltage distribution on the positive pole or the negative pole is shown in Figure 3.

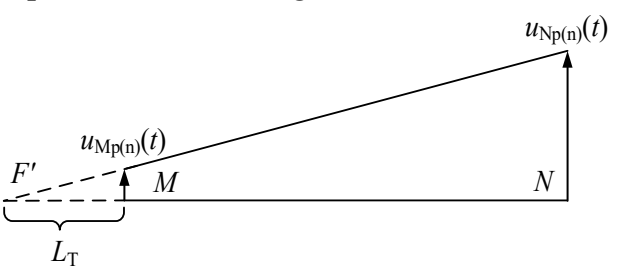

Figure 3. Voltage distribution of the HVDC lines under external faults

In Figure $3, F^{\prime}$ is the virtual fault point. The relationship between the filtered voltage and current will satisfy (11):

$$
u_{\mathrm{Mp}(\mathrm{n})}(t)=\left[r_{s} \cdot i_{\mathrm{Mp}(\mathrm{n})}^{\prime}(t)+l_{s} \frac{\mathrm{d} i_{\mathrm{Mp}(\mathrm{n})}^{\prime}(t)}{\mathrm{d} t}+r_{m} \cdot i_{\mathrm{Mn}(\mathrm{p})}^{\prime}(t)+l_{m} \frac{\mathrm{d} i_{\mathrm{Mn}(\mathrm{p})}^{\prime}(t)}{\mathrm{d} t}\right] \cdot L_{\mathrm{T}}+u_{\mathrm{Np}(\mathrm{n})}(t)
$$

According to (11), the calculated fault distance of the positive pole and the negative pole will be the distance between $M$ and $F^{\prime}$. It is easy to know from Figure. 3 that when an external fault occurs, the calculated $L_{\mathrm{T}}$ will be greater than the line length $L$ or less than 0 .

In summary, when the metallic PTP or NTP fault occurs inside the DC line, the calculated fault distance $L_{\mathrm{T}}$ of the fault pole will be stable between $0 \sim L$; when the PTP fault occurs inside the DC line, the fault distance $L_{\mathrm{T}}$ calculated by 1 -mode electrical quantities will be stable between $0 \sim L$; when the external fault occurs, the calculated fault distance $L_{\mathrm{T}}$ of the positive pole and the negative pole will be greater than the line length $L$ or less than 0 .

\section{Novel Two-step Distance Protection Scheme for flexible HVDC Transmission Lines}

When calculating the fault distance, the fault point voltage used in (5) should be the filtered fault point voltage, which can not drop to zero immediately and make it difficult to calculate the fault distance accurately. With the decrease of the cut-off frequency, on the one hand, the time for the fault point voltage to drop to zero after filtering will increase, so the time for calculating the fault distance will increase; On the other hand, the error of equivalent $\pi$ model circuit will be reduced, and the accuracy of calculating the fault distance will be improved. To balance the speed and reliability, the novel distance protection is set as a two-step distance protection. 
Distance relay Zone-I uses the filter with a higher cutoff frequency $f_{\mathrm{c} 1}$ to detects the nearby fault rapidly; while distance relay Zone-II uses the filter with a lower cut-off frequency $f_{\mathrm{c} 2}$ to detects the remote fault reliably. Because the cut-off frequency $f_{\mathrm{c} 2}$ used in distance relay Zone-II is lower, the time required for calculating the fault distance is longer than that of Zone-I.

The criterion of the distance relay Zone-I is constructed in (12):

$$
\left\{\begin{array}{l}
\text { criterion-1:0 }<L_{\mathrm{T}_{-} \mathrm{I}}<L_{\text {set1 }} \\
\text { criterion-2:0 } 0 L_{\mathrm{Tp}_{-} \mathrm{I}}<L_{\text {set1 }} \\
\text { criterion-3:0 } 0 L_{\mathrm{Tn}_{-} \mathrm{I}}<L_{\text {set1 }}
\end{array}\right.
$$

The criterion of the distance relay Zone-II is constructed in (13):

$$
\left\{\begin{array}{l}
\text { criterion-1:0 }<L_{\mathrm{T}_{-} \mathrm{II}}<L_{\mathrm{set} 2} \\
\text { criterion-2:0 } 0 L_{\mathrm{Tp} \_ \text {II }}<L_{\mathrm{set} 2} \\
\text { criterion-3:0 } 0 L_{\mathrm{Tn} \text { II }}<L_{\mathrm{set} 2}
\end{array}\right.
$$

Where $L_{\text {set1 }}$ and $L_{\text {set2 }}$ is the setting value of the distance relay Zone-I and Zone-II. When the consecutive N-times calculated results satisfy criterion-1 in (12) or (13), criterion-1 is thought to be satisfied; criterion-2 and criterion-3 in (12) or (13) are satisfied with the same conditions. If criterion- 1 is satisfied, or criterion- 2 and criterion-3 are satisfied at the same time, the fault will be judged as a PTP fault; if only criterion-2 is satisfied, the fault will be judged as a PTG fault; if only criterion-3 is satisfied, the fault will be judged as an NTG fault.

\section{Simulation Analysis}

\subsection{Simulation model of flexible HVDC project}

According to Figure 4, a 500-kV typical topology of fourterminal half-bridge MMC based DC grid is established on the PSCAD/EMTDC platform. The system adopts the symmetric monopole structure and the overhead lines adopt the frequency-dependent model. The length of Line 1 is $200 \mathrm{~km}$, the fault occurrence time is $1 \mathrm{~s}$, and the sampling rate is set to $5 \mathrm{kHz}$. The cut-off frequency $f_{\mathrm{c} 1}$ of distance relay Zone-I is set as $50 \mathrm{~Hz}$, and the cut-off frequency $f_{\mathrm{c} 2}$ of distance relay Zone-II is set as $20 \mathrm{~Hz}$. The setting value of distance relay Zone-I is $60 \mathrm{~km}$, and the setting value of distance relay Zone-II is $160 \mathrm{~km}$. When consecutive 5-times calculated fault distances meet the action judgment, the protection will act.

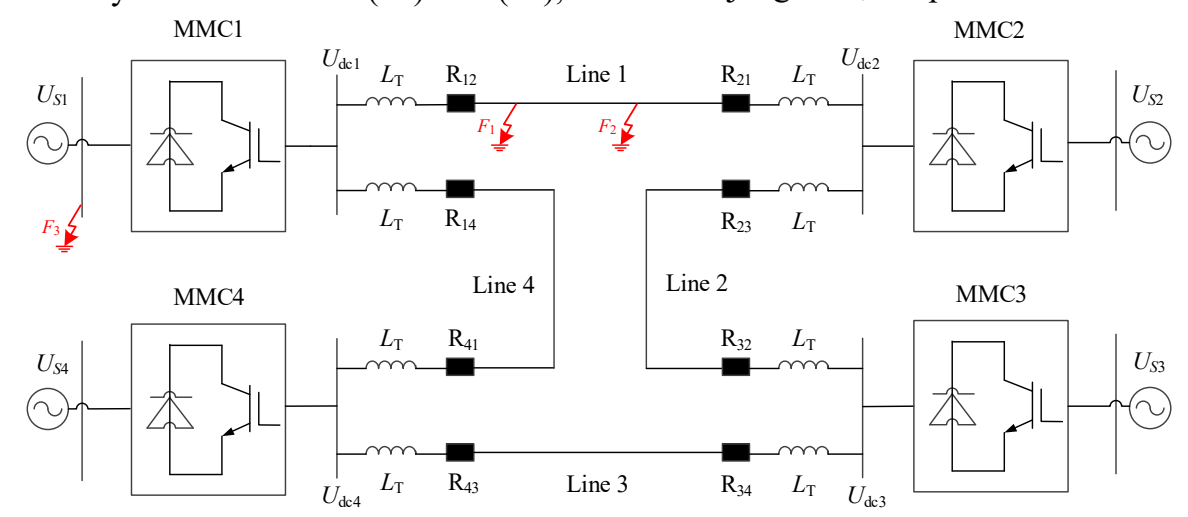

Figure 4. Typical topology of four-terminal half-bridge MMC based DC grid

\subsection{Discussion on simulation results of typical working conditions}

5.2.1 Working conditions of typical internal faults. When the metal PTG fault $F_{1}$ occurs at the place $20 \mathrm{~km}$ away from $\mathrm{R}_{12}$, the simulation results of the two-step distance protection are shown in Figure 5. As shown in
Figure 5(a), after the fault occurs, the fault distance $L_{\mathrm{TP}_{-} \mathrm{I}}$ gets into the action interval $0 \sim \mathrm{L}_{\text {setl }}$ at $8 \mathrm{~ms}$, and the calculated $L_{\mathrm{TP} \_}$equals $19.85 \mathrm{~km}$ after $45 \mathrm{~ms}$ of fault occurrence, which is close to the real fault distance; As shown in Figure 5(b), The calculated fault distance of the negative pole is not stable in the action interval. Therefore, the two-step distance protection can reliably identify the fault as a PTG fault after $8 \mathrm{~ms}$. 

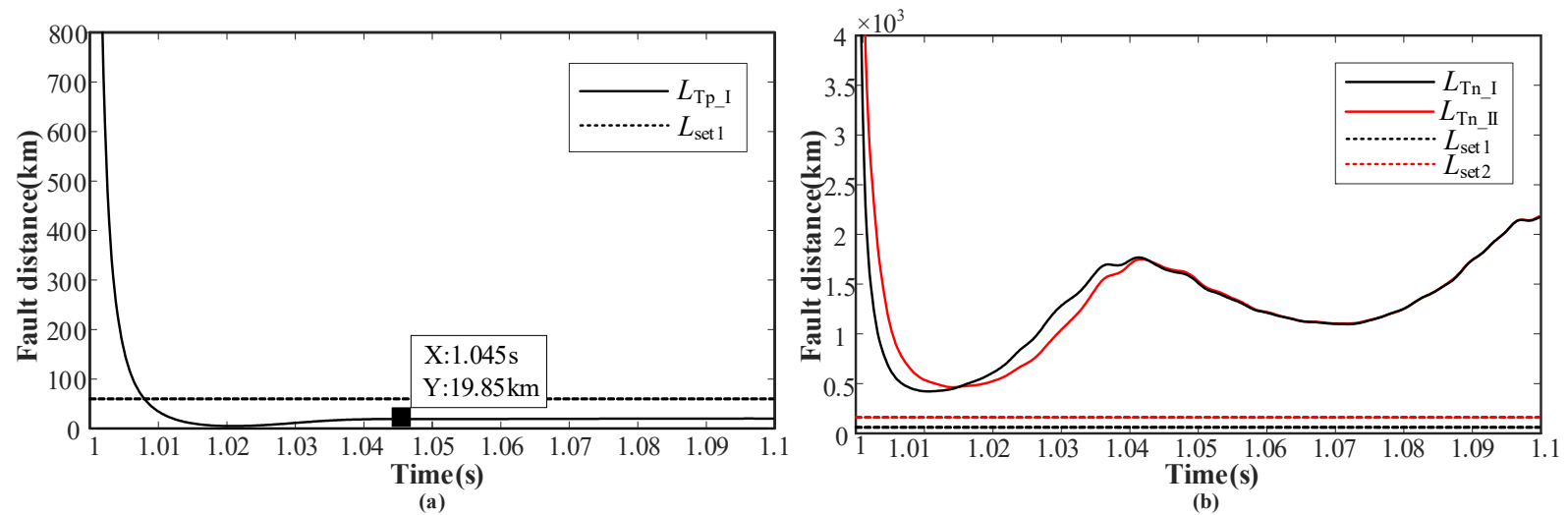

Figure 5. Simulation results of protections on Line1 when a fault occurs on the positive line at a distance of $20 \mathrm{~km}$ from $\mathrm{R}_{12}$. (a) The fault line (b) The non-fault line

When the metal NTG fault $F_{2}$ occurs at the place $140 \mathrm{~km}$ away from $R_{12}$, the simulation results are shown in Figure 6. As shown in Figure 6(a), the fault distance calculated by the two-step distance protection of the positive pole is not stable in the action interval. As shown in Figure 6(b), the calculated fault distance $L_{\mathrm{TN} \_}$ does not gets into the action interval $0 \sim L_{\text {set1 }}$. The

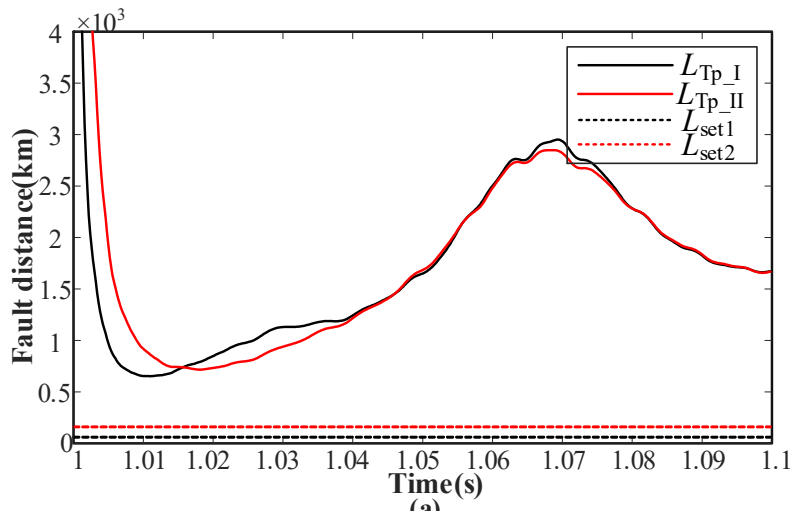

(a) calculated fault distance $L_{\mathrm{TN} \text { II }}$ is relatively stable and it gets into the action interval $0 \sim L_{\mathrm{set} 2}$ at $25 \mathrm{~ms}$. The calculated fault distance $L_{\mathrm{TN} \_ \text {II }}$ equals $139.8 \mathrm{~km}$ after $65 \mathrm{~ms}$ of fault occurrence, which is close to the real fault distance. Therefore, the two-step distance protection can reliably identify the fault as an NTG fault after $25 \mathrm{~ms}$.

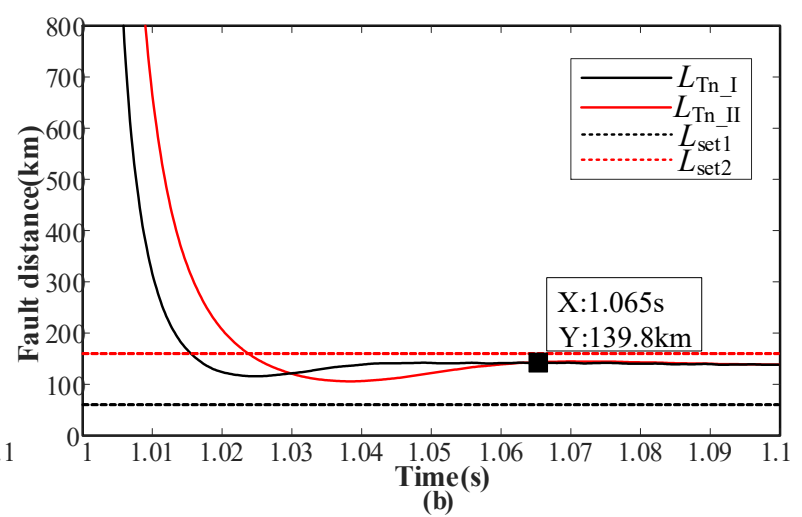

Figure 6. Simulation results of protections on Linel when a fault occurs on the negative line at a distance of $140 \mathrm{~km}$ from $\mathrm{R}_{12}$. (a) The fault line (b) The non-fault line

5.2.2 Working conditions of typical external faults. When three-phase short-circuit fault $F_{3}$ occurs on the AC bus at the rectifier side, simulation results of the two-step distance protection are shown in Figure 7. As shown in Figure 7, after the fault occurs, the fault distance $L_{\mathrm{T}_{-} \mathrm{I}}$ and $L_{T_{\text {II }}}$ calculated by 1 -mode electrical quantities are always less than 0 . At the same time, according to the simulation results, the calculated fault distance of the positive pole and negative pole are also not stable in the action interval. In this case, the two-stage distance protection will not operate reliably.

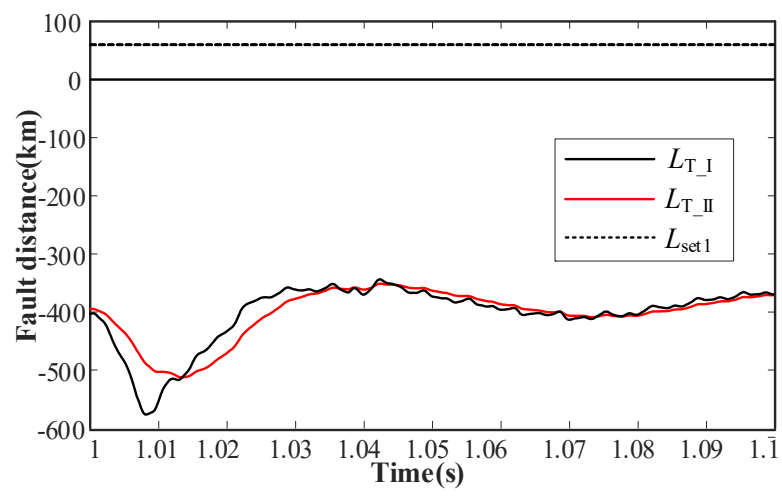

Figure 7. Simulation results of protections on Linel when a fault occurs on the AC bus

\subsection{Comprehensive simulation analysis}

Considering different fault conditions, the performance of the two-step distance protection is verified by 
comprehensive simulation. Some simulation results are shown in Table 1.

Table 1. Simulation results of distance protection when various PTG and PTP faults occur

\begin{tabular}{ccccccccccc}
\hline Fault type & PTG & PTG & PTG & NTG & NTG & \multicolumn{7}{c}{ PTP } \\
\hline $\begin{array}{c}\text { Fault } \\
\text { position }\end{array}$ & $20 \%$ & $40 \%$ & $60 \%$ & $80 \%$ & $100 \%$ & $20 \%$ & $40 \%$ & $60 \%$ & $80 \%$ & $100 \%$ \\
\hline $\begin{array}{c}\text { Transition } \\
\text { resistance }\end{array}$ & \multicolumn{1}{c}{ Operation time(ms)/Fault pole selection } \\
0 & $10.5 / \mathrm{P}$ & $18.5 / \mathrm{P}$ & $23.5 / \mathrm{P}$ & $28.0 / \mathrm{N}$ & $\mathrm{NO}$ & $10.5 / \mathrm{B}$ & $19.0 / \mathrm{B}$ & $22.5 / \mathrm{B}$ & $28.0 / \mathrm{B}$ & NO \\
$20 \Omega$ & $18.5 / \mathrm{P}$ & $29.5 / \mathrm{P}$ & $33.0 / \mathrm{P}$ & $37.5 / \mathrm{N}$ & $\mathrm{NO}$ & $/$ & $/$ & $/$ & $/$ & $/$ \\
$50 \Omega$ & $\mathrm{NO}$ & NO & NO & NO & NO & $/$ & $/$ & $/$ & $/$ & $/$ \\
\hline
\end{tabular}

It can be seen from Table 1 that when the metallic PTG, NTG and PTP faults occur in the protection area $(0 \sim 0.8 L)$, the two-step distance protection can operate correctly and select the fault pole. When the pole-to-ground fault with $20 \Omega$ transition resistance occurs in the protection area, the two-step distance protection can operate reliably; when

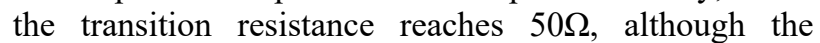
protection can not operate, the protection will not maloperate when the fault occurs at the end of the line. Therefore, the novel two-step distance protection has good selectivity.

\section{Conclusion}

Aiming at the problems of the existing main protection and backup protection of the HVDC lines, a novel distance protection scheme for flexible HVDC transmission lines which has features of both fast operation speed and high sensitivity is proposed in this paper. Based on the lumped parameter model of DC lines at low frequency, an iterative calculation method of fault distance is proposed, which can eliminate the influence of distributed capacitive current. To improve the operation speed and reliability of the distance protection, a two-step distance protection is proposed. Distance relay Zone-I uses the filter with a higher cut-off frequency $f_{\mathrm{c} 1}$ to detect the nearby fault rapidly; while distance relay Zone-II uses the filter with a lower cut-off frequency $f_{\mathrm{c} 2}$ to detect the remote fault reliably. Relative simulations prove that the novel twostep distance protection can respond to metallic pole-toground faults and pole-to-pole faults rapidly and reliably.

\section{Acknowledgments}

This work was supported in part by the key project of smart grid technology and equipment of National Key R\&D Program of China (2016YFB0900600), and in part by the National Natural Science Foundation of China (51477061).

\section{References}

1. Tang G.F., Luo X., Wei X.G. (2013) Multiterminal HVDC and DC-grid technology. J. Proceedings of the CSEE, 33: 8-17.

2. Zhong Q., Tong J.F., MA X.H. (2016) Deterministic design of LCL filter for voltage source converter. J. High Voltage Engineering, 42: 3068-3074.

3. Ahmed N., Angquist L., Norrga S., et al. (2014) A computationally efficient continuous model for the modular multilevel converter. J. IEEE Journal of Emerging \& Selected Topics in Power Electronics, 2: 1139-1148.

4. Tang G.F., He Z.Y., Peng H., (2016) Discussion on Applying the VSC-HVDC Technology in Global Energy Interconnection. J. Global Energy Interconnection, 4: 116-123.

5. Liang Z.F., Dong Y., Zhang Z.G., (2014) Statistical analysis on forced outages of HVDC transmission systems in state grid corporation of China from 2006 to 2012. J. Automation of Electric Power Systems, 38: $1-5$.

6. Cao J.F., Wang G., Zhang H.F., (2008) Study on line protection configuration and optimization of HVDC line. J. Southern Power System Technology, 2: 83107.

7. Suonan J.L., Hou Z., Song G.B., et al. (2011) Distance protection for HVDC transmission line based on distributed parameter model. J. Automation of Electric Power Systems, 35: 53-57.

8. Suonan J.L., Zhang J.K., Jiao Z.B., et al. (2013) Distance protection for HVDC transmission lines considering frequency-dependent parameters. J. IEEE Transactions on Power Delivery, 28: 723-732.

9. Qin Y., Wen M.H., Zheng J.C., et al. (2018) A novel distance protection scheme for HVDC lines based on R-L model. J. International Journal of Electrical Power \& Energy Systems, 100: 167-177.

10. Qin Y., Wen M.H., Bai Y., et al. (2020) A Novel Distance Protection Scheme for HVDC Transmission Lines Based on Criterion Extremum Online Estimation. J. International Journal of Electrical Power \& Energy Systems, 40: 3888-3896. 